\title{
Methods to design bespoke sockets
}

The socket is an integral and important part of the prosthetic limb, providing the link between the body and technology. The quality of this connection must be considered as we strive towards embodiment of the prosthetic limb. The purpose of the socket is not just to provide a container for the residual limb, but to provide a vessel where biomechanical forces can be transmitted from the body to the prosthetic componentry in the most energy efficient manner, whilst protecting the underlying tissues. The residual limb consists of bone surrounded by an envelope of soft tissues, including muscles which may no longer have insertion points. As the bone is able to move within the envelope of soft tissues, poor transmission of force and discomfort within the prosthesis can occur. Stabilising tissues to minimise bone movement within the socket is considered one of the primary goals in designing and constructing a well-fitting socket.

\section{What makes a good socket?}

It is difficult to define what makes a good socket. Factors such as comfort are often used to determine the success of a socket. This subjective measure however is difficult to quantify and a person's perception of comfort can change day-to-day according to how the wearer is feeling or what tasks are being performed. In addition to comfort, prosthetists consider factors such as the volume of the socket, preserving range of motion of intact joints and quality of suspension when determining if the fit and design of the socket is appropriate. The final judgement however always belongs to the wearer as a prosthetic limb must be comfortable and useful if it is to used regularly. Whilst it is difficult to quantify what makes a good socket we do know that prosthetic limb wearers are less likely to use a prosthesis with an ill-fitting socket. Socket discomfort is frequently reported as a compounding factor for prosthesis rejection. Other socket related factors which contribute to limb abandonment include weight, heat, difficulty donning and poor suspension $\left(^{1-5}\right)$.

Every person who wears a prosthetic arm is unique, and therefore the prosthetic socket must be custom made to meet the individual requirements of the wearer. Design criteria will be established following an assessment of the wearer's anatomy, needs and preferences.

The health and condition of the residual limb depends greatly on the surgery which was performed and the person's recovery. Poor surgical technique or post-amputation complications can result in socket-fitting issues later. Ideally the amputation should result in a mid-length residual limb (1/3 to $1 / 2$ of the original bone length). A mid-length residual limb is preferable when limb-fitting as the limb will have a long enough lever arm and muscle strength to allow good powerful movement within a prosthesis, whilst having space for prosthetic componentry to be fitted without affecting overall limb length. Maintaining overall limb length is important for cosmesis, body symmetry and proprioception. Complications which may occur following amputation surgery include bony spurs, nerve pain, muscle imbalance and loose tissue. These would be identified during the patient assessment process conducted by the prosthetist and must be considered when designing an appropriate socket.

\section{Socket design considerations}

The prosthetic socket is a custom-made product and should be designed by a competent person, namely a prosthetist, following a medical history and assessment of the user. The exact design of 
the socket will be influenced by factors such as skin quality, scarring, what the prosthesis will be used for, and how it will be powered. When designing a socket there are a number of key features which must be considered. These features cannot be considered individually as each feature will influence the others;
a. The tightness of the socket (socket fit)
b. Stability within the socket
c. How the socket will be held on (suspension)
d. How high the socket will extend on the arm (trimlines)
e. How easy the socket will be to don/doff
f. What materials the socket will be made from

\section{a. Socket fit}

When we discuss socket biomechanics, stiffest path principle and energy efficiency, we normally assume that the resultant socket will be tight-fitting. However the human body is subject to volume changes and these will affect the tightness of the prosthetic socket on the residual limb. Whilst volume fluctuations are not as troublous in the upper limbs as the lower limbs, prosthetic limb wearers will notice variations in socket fit between morning and evening, from day to day and over time. Traditionally, wearers of cosmetic and body powered prostheses have used stump socks to manage any changes in volume. Stump socks are tubular shaped socks similar to those worn on the feet. They are available in various lengths and thicknesses, and users will often wear socks in layers, adding and removing them to accommodate volume fluctuations. As well as reducing the biomechanical efficiency of the socket by introducing compressible layers, the practice of using multiple socks under the socket can increase skin temperature and bulkiness. Socket volume cannot be managed in this way within a myoelectric prosthesis, as the electrodes need to be in direct contact with the skin. There is a growing trend for sockets with adjustable volume. These designs often incorporate a clamshell construction with a dial adjustable lacing system, such as a Boa device. Advantages of such systems are that small changes in volume can be accommodated, and donning and doffing the socket is easier, however the socket will be bulkier and care must be taken to ensure that the shape matching aspects of the socket, and electrode positions, are not compromised.

\section{b. Stability}

The socket provides the physical and mechanical link between the body and the prosthetic componentry. During limb movement the remaining bone will move within its envelope of soft tissue. In order to transmit forces effectively it is essential that the soft tissues of the residual limb are stabilised as this will minimise unwanted bone and tissue movement. Internal shear forces, caused by longitudinal displacement or transverse and rotational displacement of tissues are known to cause damage to tissue. To minimise internal shear forces it is important that the socket environment provides a stiff coupling between the bone and the tissues. This will help ensure that there is minimal pistoning both within the tissues of the limb, and between the socket and the skin. Vulnerable areas of the residual limb, such as the tissues around the scar or those stretched over the cut end of bone, are less likely to breakdown in sockets which adhere to the stiff coupling principle ${ }^{6}{ }^{6}$. Pistoning between the socket and skin is exasperated by the inclusion of heavy componentry within the limb build. Reducing pistoning must be a priority when designing a socket and can be managed by incorporating a suitable suspension system. 
Soft tissues are at higher risk of breakdown if they are subjected to high pressures applied over a prolonged period. Studies have shown that pressures should not exceed $15-20 \mathrm{mmHg}$ for extended periods of time $\left({ }^{7}\right)$. Whilst pressures inside a prosthetic socket often exceed this level, the risk is minimised by wearing time. This is well documented in lower limb prosthetic sockets, where pressure is off-loaded by the cyclic loading during the gait cycle $(7,8)$, but remains an underexplored area within upper limb prosthetic design. Reducing shear pressure is critical for preventing deep tissue injury ${ }^{9,10}$. One method which prosthetists use to reduce socket pressures is to apply the principle of total contact socket design. In essence, this is where forces are spread over as large a surface area as possible based on the formula Pressure=Force/Area. Surface matching is critical in avoiding high pressures over bony prominences and associated boundary pressure gradients.

Contouring the socket, through either surface matching to the underlying bony anatomy or by compressing soft tissues will help prevent rotation of the socket and subsequent skin damage caused by torsional forces. Rotation of sockets is commonly an issue when trimlines are kept low, for example in trans-radial sockets which do not extend over the elbow and in under-the-shoulder trans-humeral designs.

A number of socket designs exist which do not adhere to the total contact principles of socket design. Instead, these have struts which make contact with the arm, providing stability for the prosthesis. Examples of these include the WILMER, Socket-less socket and Hi-Fi socket.

\section{c. Suspension}

A good socket should not piston on the arm whilst being worn. During lifting the additional weight of the object being carried may cause the prosthetic arm to slip distally. In addition, during flexion and extension of the arm, a prosthetic socket which is not adequately anchored can move on the soft tissues causing friction and potential skin breakdown. To avoid slippage it is essential that the socket is securely suspended on to the arm. Unless an osseointegration procedure has been performed most sockets will utilise either skin traction, an indirect skeletal suspension method, or harnessing to suspend the prosthesis to the body. Harnessing is the method of attaching the prosthesis to the body using straps. Normally two or more straps will be attached to the socket, then the strapping will run up the arm, across the back and under the axilla of the other arm. Buckles or Velcro may be included to allow the wearer some adjustability for bulky or light-weight clothing. Whilst harnessing can be used for proximal levels of amputation it is rarely used with below elbow prostheses as the straps can be cumbersome for the wearer. In addition, the harnessing process is time-consuming, strapping stretches over time and is difficult to keep clean. Issues with harnessing are reported as reasons for limb abandonment.

Insert figure 1.

Examples of harnessing

In-direct skeletal suspension is where the socket or an accessory is shaped to resist slippage. Shaping the socket so that it is narrow above a bony prominence will provide a resistance to downwards slippage. There are many methods of in-direct skeletal suspension that can be used, whilst some rely solely on surface matching of the underlying bony anatomy, others methods apply forces to soft 
tissue above the bony prominence to enhance the effect. This technique can be incorporated within the design of the socket itself, such as in a supracondylar or supra-olecranon styled socket, or provided within an accessory such as a humeral cuff. To enable easy donning of the socket, trimlines, material construction will need to be considered.

Cuff suspension, a form of in-direct skeletal suspension where the prosthesis is suspended by an adjustable circumferential force proximal to the socket used to be a common prescription. It is no longer frequently used in clinical prosthetic designs but features within many open-source socket designs within the additive manufacture community.

In disarticulations, indirect skeletal suspension can often be achieved through surface matching around the distal condyles. This is beneficial as it means that the socket does not need to extend to the proximal joint, but leads to issues donning and doffing the socket.

Fleshy residual limbs can be suspended using skin traction, with or without a suction valve or vacuum. These sockets are normally very tight and the wearer may need to use a sleeve to pull their tissues into the socket. Additional shaping can be applied around the proximal trimline to mould the socket over the anatomy to provide additional in-direct skeletal suspension.

As with lower limb prosthetic sockets, a silicone or gel suspension liner can be used as the suspension component. The liner has a high coefficient of friction which means that it sticks firmly to the skin unless it is rolled off. The liner is rolled onto the skin then the socket is donned over the top. The socket will connect to the liner by either a lanyard, pin and lock system or a vacuum. Liners provide a secure method of suspension providing that an appropriate sized liner has been selected. A limited size range of liners is available from commercial suppliers but they also can be custom made in prosthetic workshops by laminating silicone over a mould of the residual limb.

Trans-humeral prosthetic sockets are normally suspended using either harnessing, vacuum suspension or a roll-on liner with lock/lanyard.

\section{d. Trimlines}

Maintaining range of motion of intact anatomical joints is a key aim in prosthetic design as any restriction in movement could lead to soft tissue contractures and/or compensatory body movements. Keeping the trimlines of the socket low will ensure that range of motion at proximal joints is not limited. It is not always possible however to keep trim lines low as objectives such as maximising surface area or providing in-direct skeletal suspension often mean that a compromise needs to be made. For example, the trimlines on a short transradial socket will normally extend above the cubital fold. This will block the amount of elbow flexion possible and unless an element of pre-flexion has been incorporated into the alignment the prosthetic user may be required to adopt compensatory movements in order to complete their activities of daily living. Full elbow extension will be restricted in most sockets which extend over the olecranon such as the supra-olecranon, brim and Muenster sockets.

It is common, especially in bilateral amputees, to remove socket material around the elbow itself. This must be accounted for prior to manufacturing the socket as cutting away material can weaken the structure of the socket. Leaving the elbow open can enhance sensation for some prosthesis wearers, for example they will be able to feel clothing through the socket. Wearers also report that their arm will be cooler if the socket has an open elbow. 
The challenges associated with determining the optimal trimline height will vary according to the physical presentation of the wearer and the design of socket being made. Trimline height is often finalised at the time of delivery of the prosthesis, and can often be altered later.

\section{e. Donning and doffing the prosthesis}

In addition to maintaining range of motion of remaining joints, the trimlines of the socket will influence how easily the prosthesis can be donned and doffed. It is important that a person with limb absence should be able to don and doff their prostheses independently. This means that the process should be simple and straightforward as it will need to be conducted one-handed, or by using residual limbs, other body segments and assistive devices if the wearer has a upper limb bilateral absence. In practice, sockets with lower trimlines will be easier to don/doff. The addition of a liner which needs to be rolled on and off, a pull-in sleeve, valve, straps, buckles etc will add complication and may be a prohibiting factor for a bilateral prosthetic user. Fitting a diagnostic socket, which is a temporary socket used by prosthetists to assess fit and suspension, can be a useful step as this will enable the wearer to practice donning and doffing the socket before the definitive is made.

Insert figure 2

A diagnostic socket being used to test socket fit, range of motion and ease of donning

\section{f. Materials for sockets}

When fabricating a socket most builds comprise of an inner socket and an outer socket. The inner socket will be formed over a modified cast or model of the residual limb and should be designed according to total contact principles. The outer socket is formed around the inner socket and connects the socket to the rest of the prosthetic limb. Channels can be built between the two sockets for cables to run through. In some cases, for example in cosmetic prosthetic limbs, the inner and outer sockets may be bonded together during fabrication to produce a more seamless cosmetic finish.

The stiffest path principle is a concept often used when designing prosthetic sockets. This means that in order to transmit biomechanics forces most efficiently, a stiff medium should be provided. For this reason, prosthetic sockets are commonly made from materials such as reinforced acrylic or polyester resin. Rigid materials however can restrict range of motion and so flexible materials are often used in areas where a stiff socket is not crucial. This can be, for example, in the wings of the socket to allow easier donning of the socket, and near the trimlines to enable a few extra degrees of movement whilst still managing soft tissue volume.

A traditional laminated socket is fabricated over a positive mould of the residual limb. Layers of fabric such as cotton, carbon fibre, fibreglass and nylon, are impregnated under vacuum with a thermosetting resin which is hardened by polymerisation. The material properties of the socket are determined by the materials used in the base lay-up, including fibre length and the direction they are orientated, and the resin matrix. By using a combination of materials within the lay-up and resins of different rigidity it is possible to create a socket with areas of stiffness and flexibility.

Insert figure 3

\section{Socket Lamination}


Compressible materials such as foams, commonly used within lower limb prosthetic sockets, are not routinely used in upper limb prosthetics. This is in part because the loads experienced by upper limbs are not as high as in the lower limb, and also because compressible materials add bulk to the socket which reduces the cosmetic appearance. Whilst foams are not commonly used, some wearers can benefit from a silicone liner or inner socket.

A roll-on silicone liner, worn next to the skin and under the socket, can protect the residual limb from shear forces and assist in scar healing. Silicone liners used along with a lanyard and cleat, or pin and lock, can provide effective suspension of the prosthesis. One advantage of using silicone sleeve suspension is that the trimlines of the socket can often be kept lower, as suspension is not reliant on the anatomy at the distal end of the bone proximal. Assuming that the trimlines are high enough to ensure suitable distribution of forces, the joint can be left free of socket material and therefore the range of motion at that joint will be optimised and a more cosmetic finish may be achieved. Roll-on silicone liners are available to purchase commercially, but in a small range of sizes. An alternative approach is to fabricate a custom roll-on liner by laminating silicone over a mould of the residual limb. Whilst this adds an additional step to the fabrication process, it is a cost effective method of incorporating a silicone liner within a prosthesis for people whose arm measurements fall outwith the commercial product range.

\section{Insert figure 4}

Examples of silicone roll-on liners: Custom made laminated liner, left. Commercial liner, right.

Another method of combining flexibility and rigidity within the socket is to fabricate a socket with a flexible inner socket and a rigid shell. Flexible materials such as EVA or silicone formed over a mould of the residual limb will produce an inner socket in which volume is contained whilst allowing flexibility at the trim lines for added comfort and range of motion. The rigid shell provides structural support and the connection to prosthetic componentry.

Socket hygiene is essential within any prescription. Through enclosing the residual limb the temperature of the skin will be increased and most wearers will experience an increase in perspiration. Excessive heat is a common complaint and reason for prosthesis abandonment. Bacteria can thrive within a prosthetic socket that is not regularly cleaned. Prosthetic sockets can be difficult to clean, especially if they contain electronic components, holes and channels. It is recommended that stump socks, if used, are replaced daily and washed in between wear. Sockets and silicone liners should be cleaned at the end of the day with an anti-bacterial wipe and left to airdry.

Consideration must be given to the quality of the material used. Only materials which are classed a medical grade and safe for human use should be used in areas of the socket which have contact with the skin that is. The European Commission and ISO Standards have produced guidance documents which classified medical devices and the materials which can be used within prosthetic sockets.

\section{Shape capture}

To create a bespoke socket, the measurements and shape of the person's residual limb must be captured. Prosthetists traditionally use a combination of measurements and a negative plaster cast 
of the limb to obtain this information, but scanning has become more popular in recent years. There is a lot of variability in scanners in relation to cost, accuracy and usability. Cheap scanners, costing a few hundred pounds, can provide reasonable images with a lower degree of accuracy. For more accurate work, for example if creating a high cosmetic finish, a more accurate scanner would be required. Tips for ensuring a good scan include adding reflective markers over anatomical landmarks. This not only assists with tracking during the scanning process itself but also helps identify the anatomical landmarks required when doing a CAD modification.

Inset figure 5

\section{Casting a transradial arm}

After the limb has been scanned the file must be uploaded to a suitable CAD package. Likewise if a plaster cast has been taken, the mould should then be filled with plaster of Paris to form a positive mould of the residual limb. This positive mould can either be scanned to make a digital image for CAD, or can be modified manually using traditional prosthetist plaster skills.

The process of modification of the image/positive model is critical for obtaining a well-fitting socket. In most cases the volume of the model must be reduced to stabilise the tissues. The amount of volume reduction required will depend on the individual's features and the type of socket being made. Reliefs over bony prominences and cut end of bones are commonly built in to the socket design. During the modification process, the trimlines of the socket should be finalised. The trimlines should be set at a height that maximises surface area, thus minimising socket forces, but allows minimal interference with range of movement, and enables the socket to be donned easily. Longer residual limbs will often require lower trimlines, particularly if the socket extends over the proximal joint. A short residual limb may require very high trimlines, to prevent the residual limb from popping out of the socket during flexion.

\section{Electrode placement}

Myoelectrodes are commonly used as a switch within myoelectric prostheses. In myo control, the electrode detects changes in the electrical activity on the skin surface caused by calcium being released prior to a muscle contraction. The electrode is normally positioned where ever the signal is strongest, normally over the muscle belly, but its position may need to be moved to avoid scar tissue or trimlines/ cut outs within the socket. Electrode placement should be determined at the time of assessment, when the socket is being planned. It is not appropriate to determine this when the wearer is not present.

\section{Task specific devices}

Success of a prosthetic limb should not be based on wearing time. When and where a person with limb absence chooses to use a prosthetic limb will vary according to the activities they are carrying out, the social environment and their limb health. It is widely acknowledged that by wearing a prosthetic socket, users will experience lowers levels of touch feedback than if they were to use their residual limb to conduct a task. This, along with the ability to grasp objects in the elbow crease is sufficient reason why many, especially congenital absence, choose either not to wear a prosthesis or to only wear one at selective times. 
For a prosthesis to be successful it must be comfortable and serve a useful purpose. That purpose may be functional or cosmetic, and it may be required daily, or less frequently. Useful might mean that it helps the wearer to perform a task, or it might fulfil a cosmetic or physiological need. Day and Wands-Silva ${ }^{11}$ found that children with congenital BE absence who have a prosthetic or assistive device are most likely to use it for bimanual tasks or for leisure activities such as sports or music. For this reason a prosthetic limb, or assistive device, could be considered as a task specific tool. Task specific tools have commonly been used with traditional body powered prostheses fitted with a wrist unit that allows the terminal device to be interchanged. This makes the prosthesis multifunctional. At times the user may choose to have a hand shaped terminal device, other times, for example when fine grasp is required he/she may select a split hook, or a passive device can be fitted such as a gripper to secure vegetables during peeling or a typing appliance. The versatility of having interchangeable terminal devices is beneficial, however it is costly to provide multiple terminal devices and can be inconvenient and sometimes awkward for the user to physically change these.

Rather than designing a multi-functional device it is often more effective to design task specific prostheses or devices. This is an area of prosthetic design that involves creativity and problem solving, and any device must be designed in consultation with the wearer to ensure a successful outcome. Non-conventional designs will require a risk assessment to be performed before delivery of the device. Children are commonly provided with cycling devices which allow them to bimanually hold the handlebars of a bicycle or scooter. Brakes are normally rerouted to be operated by the sound side. A feature of this device is that the user must be able to detach themselves from the bike quickly. This is important to prevent serious injury if the child were to fall off the bike, as it may be dangerous for them to physically be attached to the bike. This can be done by either designing a device that can be detached quickly for the handlebars, or by the user pulling themselves free from the device. Another area where children will commonly use a device is when learning a musical instrument. Music is known to have therapeutic benefits and is included in the national curriculum. Exclusion from such activities would be detrimental to the child. Musical devices are normally designed on a bespoke case. A simple socket or band is usually made with an attachment to either hold, support or operate the instrument. Devices like these are likely to be worn every week during lessons, but may be abandoned once the child has moved on to a new hobby. For this reason, a low cost built may be appropriate, but as with all assistive devices, quality must not be compromised as this could affect the childs ability to progress. The strength of the device must be considered as a prosthesis or assistive device that breaks is not useful, and may prevent the child from participating in tasks. There are many open source 3D printed patterns available on the internet. Some of these might be suitable for task specific activities, but the fit, strength of the device should be considered before it is provided.

\section{Common transradial socket designs}

Trans-radial prostheses are the most commonly prescribed upper limb prosthetic device ${ }^{12}$. There are many sockets designs suitable for residual limbs at this level, and selection of which type to use will be determined following a full patient assessment.

When fitting a short fleshy residual limb, the socket must encompass a large section of the arm, commonly extending to the elbow crease or beyond, to maximise surface area. Many people who have short fleshy residual limbs will have underdeveloped skeletal anatomy, and length discrepancies may be present if the limb was absent at birth or amputated in childhood. Sockets for this level can be designed to worn either directly next to the skin, or with an interface liner or sock. 
The Muenster socket was created by Hepp and Kuhn in the 1950s for persons with short residual limbs and is instantly recognisable due to a convexity on the anterior trim. This is a tight-fitting selfsuspending socket with high trimlines to enclose all tissues below the elbow. An anterior-posterior compression force is applied to the triceps tendon and either side of the biceps tendon. This creates a relief for the biceps tendon and the Muenster socket iconic appearance. The socket has high trimlines and as a result both flexion and extension of the elbow will be limited. Prosthetists commonly compensate for this reduction in elbow flexion by pre-flexing the alignment of components to ensure that the prosthesis remains functional for body-centred activities.

The Brim socket is a tight-fitting socket that relies on skin traction for suspension. Additional suspension is provided by a circumferential shallow grove which is cut into the plaster model at the level above the humeral condyles during cast modification. This grove provides minimal indirect skeletal suspension in people with underdeveloped condyles, but when incorporated within a tightfitting socket increases the skin traction. Like other sockets designed for short fleshy residual limbs, the brim socket restricts elbow flexion and extension due to its high trimlines.

Anatomically contoured interface (TRAC) is an aggressively modified hybrid of the Muenster and Northwestern sockets. This design requires a flexible inner socket. The socket is contoured in five areas to stabilze muscle groups and prevent rotation of the socket.

\section{Insert figure 6}

Examples of SSOS, left; Muenster socket, centre; Brim socket plaster cast, right

When considering a socket design for a mid-length residual limb there are many tried and tested socket designs to choose from. Selection will normally be determined by suspension type and prosthetist preference. In these designs the trimlines are lower anteriorly, allowing more normal elbow flexion.

The Northwestern socket is perhaps the most famous transradial socket design. Created by John Billock at Northwestern University in the 1970s, this socket utilises a tight medio-lateral diameter fit over the humeral epicondyles for suspension. This is accompanied by a low anterior trimline, extending up to $45 \%$ of the overall length of the socket. Due to the tight $\mathrm{M} / \mathrm{L}$ dimension of the design, a progressive wear program may be required to build up tolerance to pressure above the condyles.

A successor of the Northwestern socket is the Strathclyde Supra Olecranon Socket (SSOS) designed by Bill Dykes at the University of Strathclyde in the 1980s. The aim was to develop a method which could be applied to most if not all trans-radial levels, allow the prosthesis to be worn as long as necessary from day one and maximise residual forearm flexion. The SSOS utilises the area above the olecranon on the triceps tendon as the main suspension area. Medial and lateral "wings" extend over the epicondyles to the parallel section of the humerus above. The primary purpose of these extensions is to keep the posterior section of the socket in place and not to provide suspension as the area above the epicondyles does not have a good tolerance to pressure. The anterior of the socket is the only trimline that varies depending on stump length.

The Otto Bock socket is a tight-fitting socket that cups the olecranon and epicondyles using a combination of indirect skeletal suspension and tissue restriction to provide suspension. Due to its intimate fit a distal pull-in tube is usually required for donning. 
The $3 / 4$ socket was developed in response to feedback from patients that their arms got too hot in the tightfitting sockets required for myoelectric control. Through an analysis of the below-elbow socket, Sauter et al determined that the proximal posterior quadrant which covers the elbow had no functional purpose, and so a socket design evolved with this area cut-away. Reported benefits include increased ventilation and improved suspension secondary to decreased perspiration.

The WILMER is an open socket design by Dick Plettenburg from Delft University. Unlike traditional total contact designs the WILMER is constructed using stainless steel tubing which is covered with a cushioned fabric. Advantages of this system are increased ventilation for the residual limb and skin contact with the external environment. Tissues however are not stabilised and stump socket forces may be higher due to the non-total-contact design.

The High-fidelity interface (Hi-Fi socket) designed by Randall Alley is a patented design based on the compression-release stabilization theory. The socket consists of three compression areas created along the shaft on the remaining bone, with gaps in between for the release of soft tissues.

\section{Conclusion}

The importance of the socket is often overlooked when considering advances in prosthetic technology. If the prosthesis is to become truly integrated into the wearer's life then the design of the socket, including how it has been fabricated must be customised to their individual needs. There may come a time soon when we are able to automate parts of the shape capture, modification and fabrication processes, but ultimately socket success will be determine the comfort and function of the device.

\section{References}

1. Davidson J. A survey of the satisfaction of upper limb amputees with their prostheses, their lifestyles, and their abilities. Journal of hand therapy : official journal of the American Society of Hand Therapists. 2002; 15: 62-70.

2. Kejlaa GH. Consumer concerns and the functional value of prostheses to upper limb amputees. Prosthetics and orthotics international. 1993; 17: 157-63.

3. Biddiss $\mathrm{E}$ and Chau T. Upper-limb prosthetics: critical factors in device abandonment. American journal of physical medicine \& rehabilitation / Association of Academic Physiatrists. 2007; 86: 977-87.

4. Kyberd PJ and Hill W. Survey of upper limb prosthesis users in Sweden, the United Kingdom and Canada. Prosthetics and orthotics international. 2011; 35: 234-41.

5. Millstein SG, Heger $\mathrm{H}$ and Hunter GA. Prosthetic use in adult upper limb amputees: a comparison of the body powered and electrically powered prostheses. Prosthetics and orthotics international. 1986; 10: 27-34.

6. Mak AF, Zhang M and Boone DA. State-of-the-art research in lower-limb prosthetic biomechanics-socket interface: a review. Journal of rehabilitation research and development. 2001; 38: 161-74.

7. Bader DL and Worsley PR. Technologies to monitor the health of loaded skin tissues. Biomed Eng Online. 2018; 17: 40-. 
8. Dumbleton T, Buis AW, McFadyen A, et al. Dynamic interface pressure distributions of two transtibial prosthetic socket concepts. Journal of rehabilitation research and development. 2009; 46: 405-15.

9. Gefen A, Farid KJ and Shaywitz I. A review of deep tissue injury development, detection, and prevention: shear savvy. Ostomy/wound management. 2013; 59: 26-35.

10. Graser M, Day S and Buis A. Exploring the role of transtibial prosthetic use in deep tissue injury development: a scoping review. BMC Biomedical Engineering. 2020; 2: 2.

11. Day S and Wands-Silva K. Opinions of paediatric upper limb amputees. MEC '14: Myoelectric Control Symposium. 2014.

12. Twiste M. Limbless statistics: a repository for quantitative information on the UK limbless population REFERRED for prosthetics treatment. Annual Report 2010-2011. Salford. 2013. 